\title{
Tratamiento de maloclusión de Clase I con discrepancia arco diente positivo
}

Treatment of class I maloclusion with positive tooth arc discrepancy

\author{
Nilo Marcial Pari Cruz ${ }^{1 a}$ \\ Wender Williams Condori Quispe ${ }^{\text {2ab }}$
}

(iD) https://orcid.org/0000-0001-8706-9526

iD https://orcid.org/0000-0002-0587-6031

Correspondencia: wcondoriq@unjbg.edu.pe

\section{Resumen}

El objetivo del reporte de caso fue evaluar el tratamiento ortodóncico de una maloclusión Clase I con discrepancia arco diente positivo en una paciente de 15 años, a quien luego de la alineación y nivelación de los arcos dentarios se le realizó tratamientos con topes de resina para levante de mordida, arcos curva reversa, arcos de retracción superior e inferior para el cierre de espacios y, finalmente, elásticos intermaxilares para una buena intercuspidación. Después de 17 meses de tratamiento se mantuvo la relación molar y canina de Clase I, se cerraron los diastemas, se logró corregir la línea media, el overjet y overbite, y se obtuvo una estética dental y facial agradable. Las diversas alternativas de tratamiento en una maloclusión Clase I dependen de factores como la severidad de la maloclusión, etiología y otros, con el fin de obtener un sistema masticatorio funcional y buena estética.

Palabras clave: maloclusión, clase I, discrepancia, cierre de espacios

\begin{abstract}
The objective of the case report was to evaluate the orthodontic treatment of a Class I malocclusion with positive tooth arch discrepancy in a 15-year-old patient, who, after alignment and leveling of the dental arches, underwent treatments, with resin stops for lifting of bite, reverse curve arches, upper and lower retraction arches for the closure of spaces and, finally, intermaxillary elastics for good intercuspation. After 17 months of treatment, the molar and canine Class I relationship was maintained, the diastemas were closed, the midline, the overjet and overbite were corrected, and a pleasant dental and facial esthetics were obtained. The various treatment alternatives in a Class I malocclusion depend on factors such as the severity of the malocclusion, etiology and others, in order to obtain a functional masticatory system and good aesthetics.
\end{abstract}

Keywords: malocclusion, class I, discrepancy, closure of spaces

\footnotetext{
${ }^{1}$ Investigador independiente. Puno, Perú.

${ }^{2}$ Universidad Nacional Jorge Basadre Grohmann. Escuela Profesional de Odontología. Tacna, Perú

${ }^{a}$ Especialista en Ortodoncia y Ortopedia maxilar

${ }^{\mathrm{b}}$ Maestro en Odontoestomatología
} 


\section{Introducción}

La estética dental es la principal razón que motiva a los pacientes a buscar ayuda de un ortodoncista, debido a que el correcto alineamiento de los dientes y una sonrisa agradable son valorados como símbolo de belleza; por el contrario, unos dientes desalineados o protruidos producen inseguridad. Es por eso que es motivo de preocupación de muchos pacientes, sobre todo de adolescentes y jóvenes, en quienes una maloclusión produce problemas psicosociales, funcionales y mayor probabilidad de sufrir traumatismos.

La maloclusión es una desarmonía entre los controles anatomofisiológicos y los segmentos dentarios del sistema estomatognático y es de etiología multifactorial. ${ }^{2}$ La más frecuente de las maloclusiones es la Clase I, que presenta una relación normal anteroposterior del maxilar con la base de la mandíbula; en lo referente a los dientes existe una oclusión entre la cúspide mesiovestibular del primer molar superior y el surco mesiovestibular del primer molar inferior. ${ }^{3}$ En relación al tratamiento, esta maloclusión, en muchos casos, solo comparte la relación molar, siendo muy variable el tratamiento debido a que se consideran diversos factores como la malposición dental, alteraciones en las relaciones verticales o transversales y otros. ${ }^{4}$

La discrepancia dentoalveolar es la desarmonía que existe entre el tamaño de las piezas dentarias y el de los maxilares, la discrepancia positiva se caracteriza porque la sumatoria de los anchos mesiodistales de los dientes es menor en relación al tamaño de los maxilares. Este tipo de discrepancia es de poca prevalencia. ${ }^{5,6}$

Las diferentes terapéuticas y, dentro de ello, la selección adecuada de una biomecánica que dé como resultado movimientos fisiológicos es el mejor plan a seguir y evitar así la recidiva; el cierre de espacios puede darse por deslizamiento o por desplazamiento de las piezas dentarias, por tal motivo implementamos un plan de tratamiento acorde al caso.

\section{Reporte de caso}

Paciente femenino, de 15 años y 1 mes. El motivo de consulta "Mis dientes están un poco chuecos". En aparente buen estado general de salud, con mucha expectativa de mejorar su sonrisa.

Al examen clínico extraoral se observó un tipo mesofacial de constitución mediana y labios competentes. En el análisis de sonrisa presentó una línea de labio superior adecuada, arco de la sonrisa no consonante, curvatura del labio superior recta, presencia de correderas bucales, sin canteo oclusal y desvío de línea media inferior $1.5 \mathrm{~mm}$ hacia la izquierda. De perfil ligeramente convexo (Figura 1).

\section{Figura 1}

Fotografías extraorales iniciales

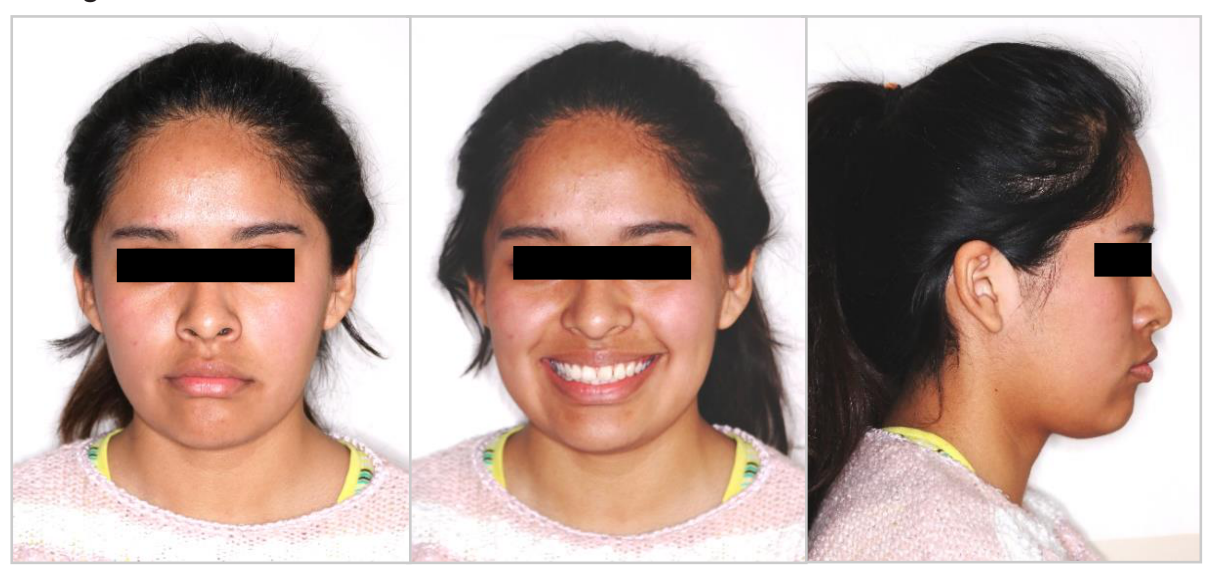


En el examen clínico intraoral, en la arcada superior, se observó una forma de arco parabólica, mesiogiroversión de las piezas 11 y 21, diastemas en las piezas 11-12, 21-22 y 22-23. En la arcada inferior también la forma del arco fue parabólico, mesiogiroversión de las piezas 31 y 41 y diastemas en las piezas 33-34, 43-44. En oclusión, la relación molar y canina derecha e izquierda fue de Clase I, overjet de $2.5 \mathrm{~mm}$, overbite de $1 \mathrm{~mm}$ (10 \%) y diastema entre las piezas 11-12, 21-22, 22-23, 34-35 y 43-44 (Figura 2).

\section{Figura 2}

\section{Fotografías intraorales iniciales}

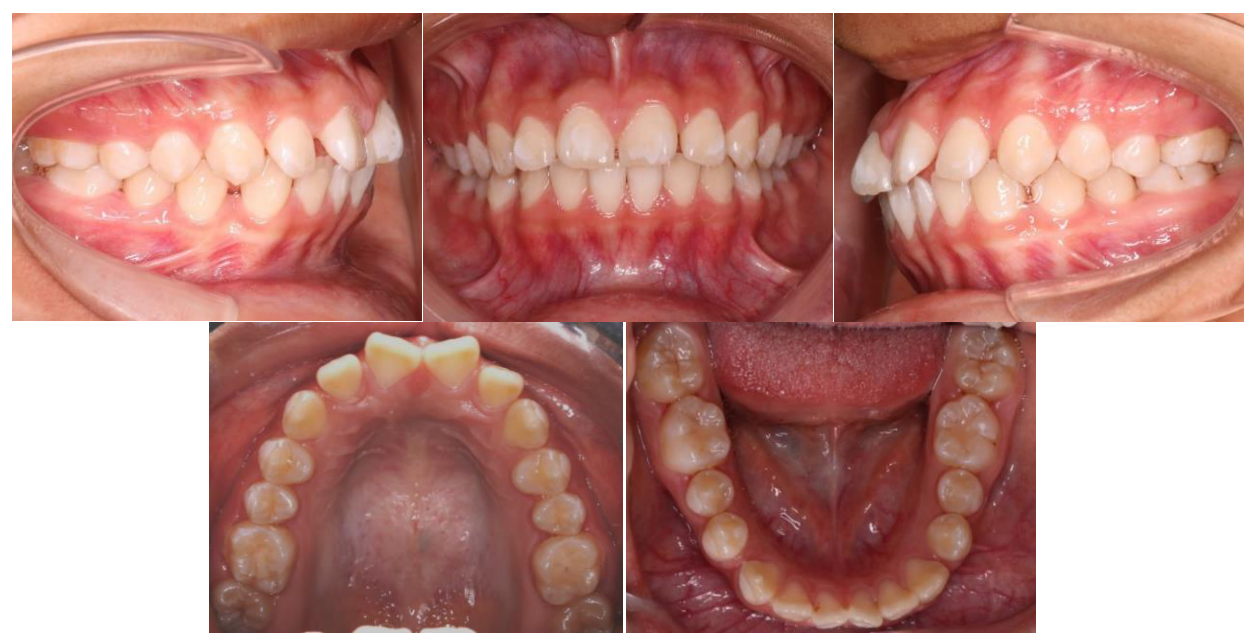

En el análisis de modelo superior, la distancia intermolar de Ricketts fue de $59 \mathrm{~mm}$ e intercanina de $37 \mathrm{~mm}$, mientras que en el modelo inferior la distancia intermolar de Ricketts fue de $54 \mathrm{~mm}$ e intercanina de $29 \mathrm{~mm}$. Presentó correspondencia molar de $42 \mathrm{~mm}$, no presentó asimetría anteroposterior ni trasversal en superior e inferior. En modelos en oclusión, la relación fue de Clase I, tanto molar como canina, el overjet de $2.5 \mathrm{~mm}$, overbite de $1 \mathrm{~mm}$, discrepancia de modelos positivo de $2 \mathrm{~mm}$, tanto en superior como en inferior y según el análisis de Bolton anterior se encontró exceso de masa dentaria inferior de $1.2 \mathrm{~mm}$ (Figura 3).

\section{Figura 3}

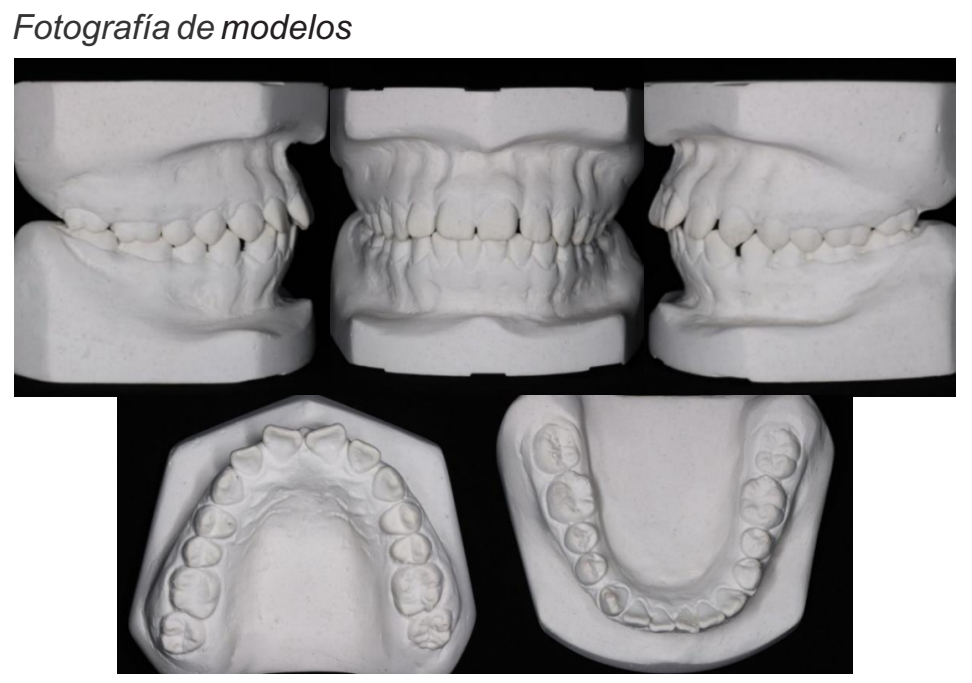


En el análisis radiográfico panorámico, los terceros molares se encontraban en evolución intraósea. Estadio Nolla 5. En el análisis cefalométrico presentó una relación esquelética de Clase I $\left(\right.$ ANB $1^{\circ}$ ), biprotrusión y proinclinación de los incisivos superiores (INA de $34^{\circ}$ y $11 \mathrm{~mm}$ ) e inferiores (INB de $37^{\circ} \mathrm{y} 9 \mathrm{~mm}$ ), el crecimiento mandibular fue normodivergente (FMA $24^{\circ}$ ), ángulo interincisivo disminuido $\left(108^{\circ}\right)$ (Figura 4).

\section{Figura 4}

Radiografía lateral inicial y cefalometría lateral inicial

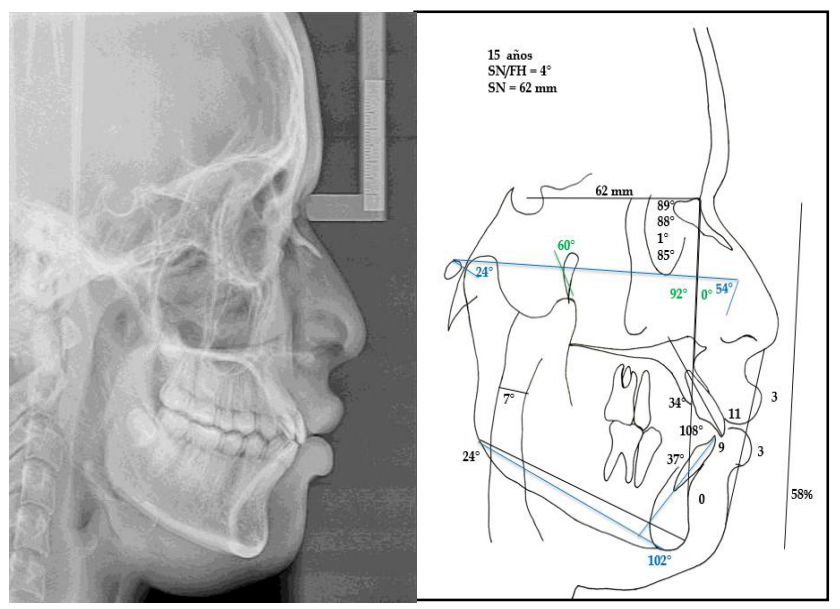

Los objetivos del tratamiento fueron corregir la guía incisiva y mantener la guía canina derecha e izquierda, mantener la Clase I esquelética, corregir la protrusión y proinclinación de los incisivos superior e inferior, corregir la mesiogiroversión de las piezas 11, 21, 31 y 41, corregir la discrepancia positiva superior e inferior y corregir la desviación de la línea media inferior.

\section{Evolución del caso}

Se inició el tratamiento ortodóncico con la instalación de la aparatología fija en dientes superiores e inferiores con brackets MBT, slot 0.022" x 0.028". En la etapa de alineación y nivelación se comenzó utilizando arcos NiTi 0.014". El control fue aproximadamente una vez al mes, se continuó el tratamiento con la secuencia de arcos hasta llegar a los arcos rectangulares. En la arcada superior se hizo un tie together en las piezas 23 a 26 , luego se procedió a cambiar el arco de acero rectangular 0.016 " x 0.022 " al arco de acero rectangular 0.017 " x 0.025 ", en la arcada inferior se volvió a recementar la banda de la pieza 46, luego se procedió a cambiar el arco de acero rectangular 0.016 " x 0.022" a uno de menor calibre de acero 0.016" y se le añadió a este arco una curva reversa (Figura 5).

\section{Figura 5}

Arco de acero rectangular en superiore inferior
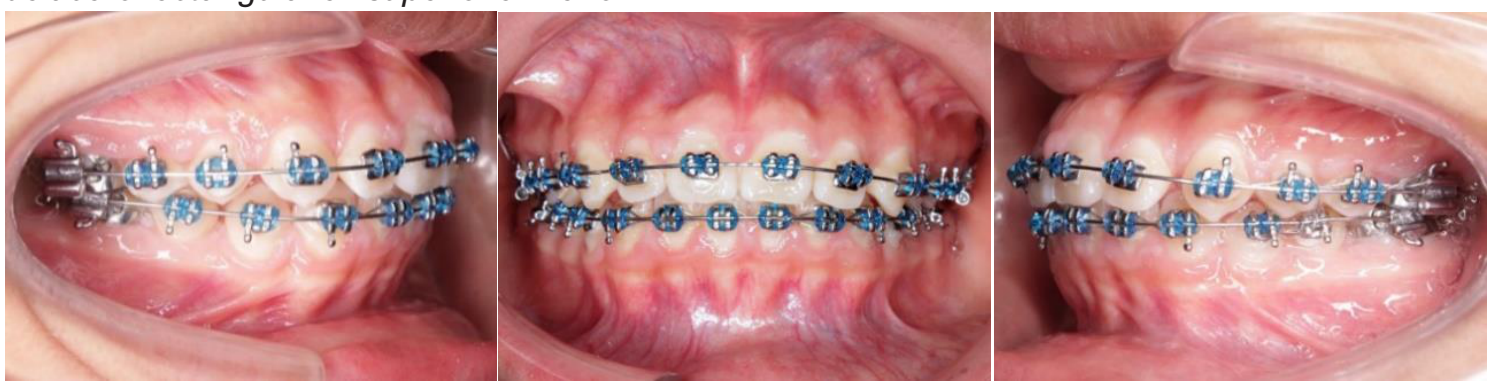
En la arcada superior se colocaron topes de resina por palatino de los caninos superiores para levantar la mordida. Para el cierre de espacio por desplazamiento se confeccionó un arco de retracción en alambre TMA 0.019" x 0.025" con step up en el segmento anterior de $0.5 \mathrm{~mm}$ para intrusión y se activó el arco $2 \mathrm{~mm}$ en cada lado; se colocó cadena elastomérica entre los incisivos superiores para cerrar los espacios en ese segmento. En la arcada inferior se colocó cadena elastomérica entre las piezas 35 y 36 para rotar la pieza 35 (Figura 6); posteriormente, en la arcada inferior se confeccionó un arco de acero inoxidable 0.018" con loops entre las piezas 33-34 y entre las piezas 43-44 para cerrar los espacios. Se retiró los topes de resina de las piezas 13 y 23; en la arcada inferior se volvió a recementar la banda de la pieza 46 y se colocó un tope de resina por oclusal de la pieza 46 para corregir el desnivel de los rebordes marginales entre las piezas 45-46, luego se cambió el arco con loops por un arco de acero recto 0.016 " con step down anterior a nivel de las piezas 33-34 y 43-44.

\section{Figura 6}

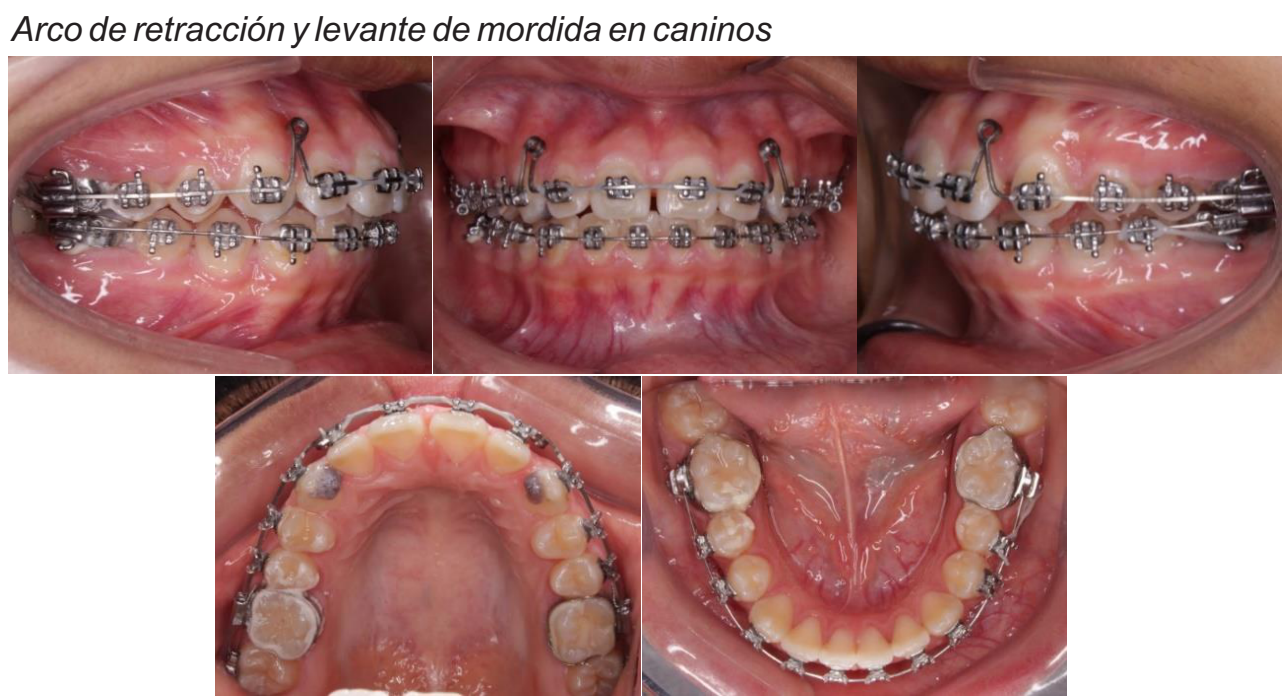

En la arcada superior se procedió a retirar el arco de retracción de TMAy, en su lugar, se colocó un arco de acero inoxidable 0.016" con dobleces para finalización. Se hizo un doblez de step down entre los incisivos centrales para la guía incisiva; en la arcada inferior se colocó un arco 0.016" con dobleces de step up en premolares derecho e izquierdo para la buena intercuspidación. Se retiró el tope de resina de pieza 46 y se usó elásticos de Clase II en izquierdo y elásticos de Clase I en derecho (Figura 7).

\section{Figura 7}

Arcos con dobleces de finalización y elásticos intermaxilares

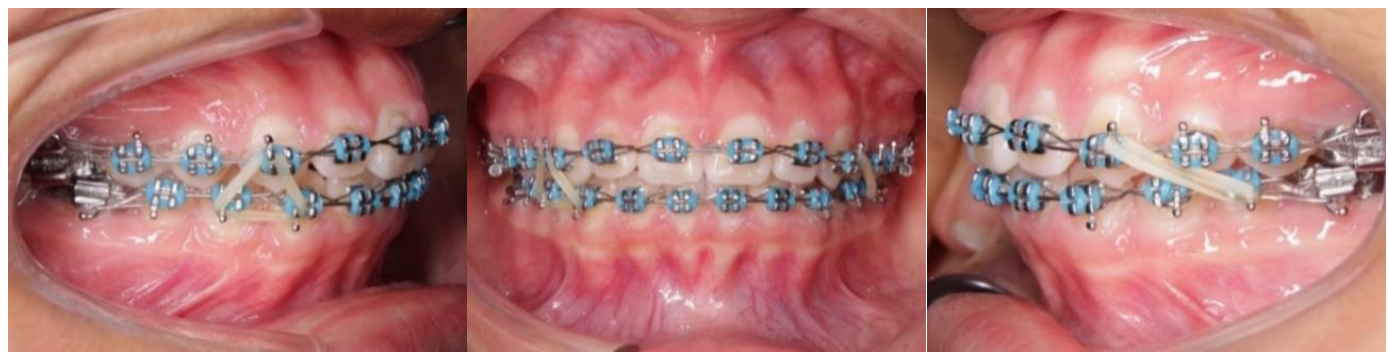


Finalmente, después de 1 año y 5 meses, cumpliendo con todos los objetivos planteados, se retiró la aparatología fija (Figura 8). Se instalaron las contenciones; en la arcada superior se colocaron 3 grapas entre las piezas 11-12, 11-21 y 21-22 para darle mayor estabilidad a ese segmento, luego se procedió a la confección e instalación de la contención removible superior tipo Wrap around, en la arcada inferior se colocó contención fija entre las piezas dentarias 34-44.

\section{Figura 8}

Fotografías intraorales de caso finalizado

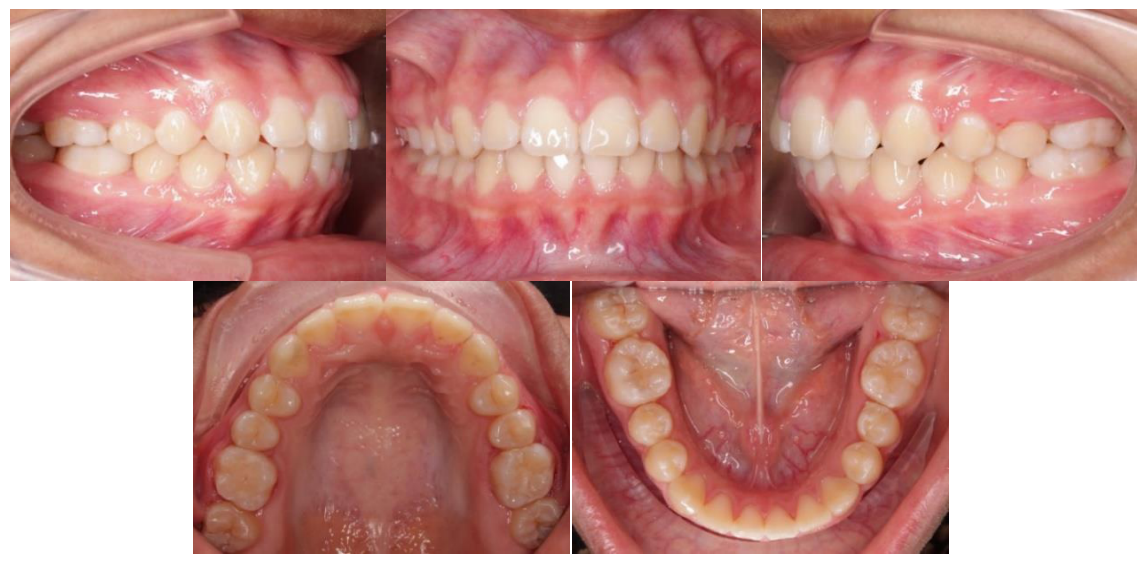

Se evaluaron las guías de la oclusión encontrándose las guías incisivas y caninas en movimientos de protrusión y lateralidad (Figura 9).

\section{Figura 9}

Movimientos protrusivos y de lateralidad

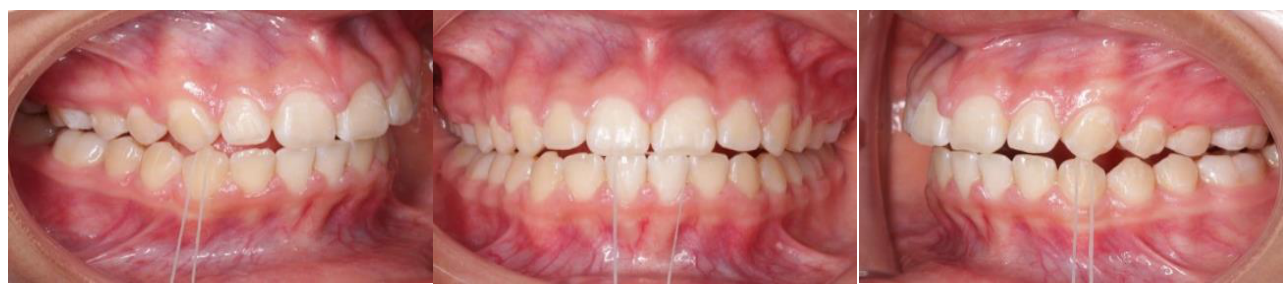

Se cumplieron con los objetivos de lograr una buena oclusión y estética corrigiendo la maloclusión que se caracterizaba por la presencia de diastemas, protrusión y proinclinación de incisivos, tanto superiores como inferiores, mesiogiroversiones y desviación de la línea media (Figuras 10).

\section{Figura 10}

Fotografías extraorales al final de tratamiento

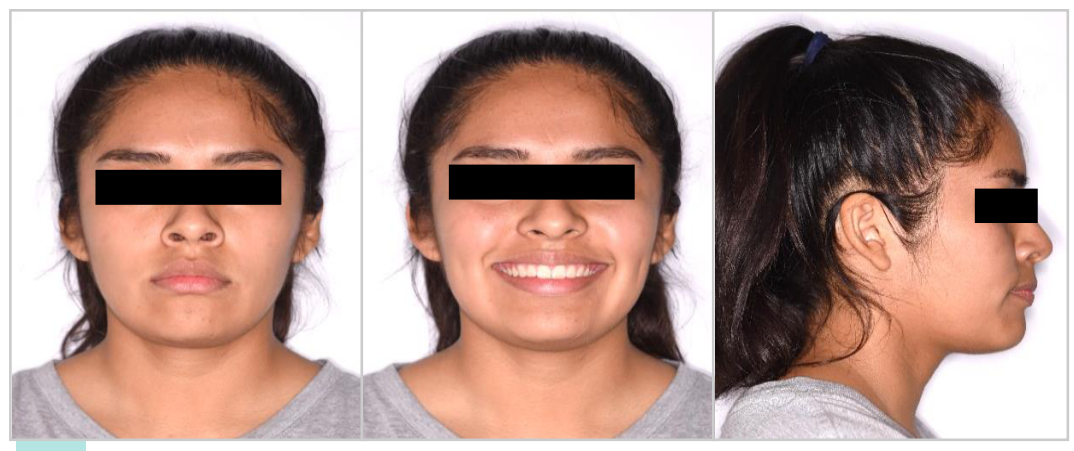


En el análisis cefalométrico final se obtuvo un ligero cambio en el ANB de $1^{\circ}$, cambió a $2^{\circ}$, disminución de la proinclinación de incisivos superiores e inferiores 1-NA de $34^{\circ}$ a $29^{\circ}, 1-\mathrm{NB}$ de $37^{\circ}$ a $33^{\circ}$, el FMA disminuyó de $24^{\circ}$ a $23^{\circ}$, manteniéndose el crecimiento normodivergente y el ángulo interincisal aumentó de $108^{\circ}$ a $120^{\circ}$ (Figura 11).

\section{Figura 11}

Análisis cefalométrico de Steiner, Tweed y Downs al inicio y final del tratamiento

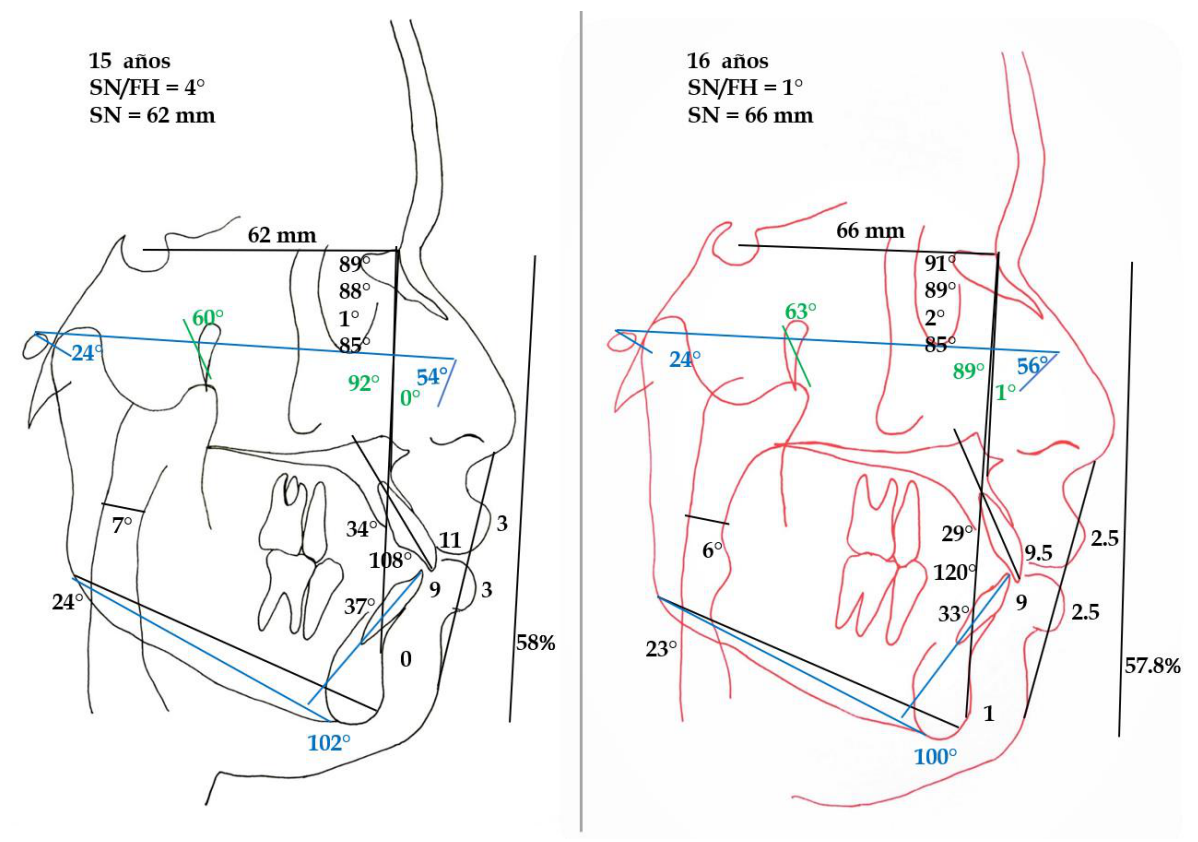

\section{Discusión}

La clasificación de Angle ${ }^{7}$ es ampliamente utilizada entre los profesionales de la Ortodoncia, debido a que es universalmente conocida. La maloclusión de Clase I de Angle es la más predominante, seguida por la Clase II y por último la Clase III. En relación al tratamiento, la maloclusión de Clase I, en muchos casos, solo comparte la relación molar, por lo tanto, el tratamiento es muy variable.,8-10

La discrepancia arco diente positiva se caracteriza por la presencia de diastemas en el arco dentario. La paciente tratada presentó discrepancia positiva de $2 \mathrm{~mm}$ en la arcada superior y $2 \mathrm{~mm}$ en la arcada inferior. Esta discrepancia tiene muy poca prevalencia en comparación con la discrepancia negativa. López, ${ }^{11}$ en un estudio realizado, encontró que solo el $8 \%$ presentaba discrepancia positiva, mientras que Huancollo ${ }^{12}$ encontró $13.6 \%$. Fernández ${ }^{13}$ encontró que el $36 \%$ tenía discrepancia positiva en el maxilar y $40 \%$ en la mandíbula.

Existen diversas alternativas de tratamiento para el cierre de diastemas; entre ellas tenemos a la mecánica por deslizamiento, en la cual, para el cierre de espacios, se utilizan cadenas elastoméricas, resortes de NiTi, hilos elásticos y elásticos intermaxilares. En nuestro caso clínico, para el cierre de espacios, recurrimos a la mecánica por desplazamiento, utilizando un arco de retracción en TMA 0.019 " x 0.025" en superior y un arco de acero con loops de retracción en inferior para controlar mejor los movimientos coronoradiculares y así tener un excelente control de la inclinación, evitando la fricción y los efectos colaterales. Santoyo y Tavira ${ }^{14}$ utilizaron para el cierre de espacio cadena elastomérica, resorte abierto, hilo elástico, arcos con ansas de contracción y 
elásticos intermaxilares de Clase II, Carrera ${ }^{15}$ y Lamas $^{16}$ usaron cadena elastomérica para cerrar diastemas. En todos los casos nombrados se obtuvieron resultados muy satisfactorios.

\section{Conclusiones}

La maloclusión Clase I se caracteriza por tener una relación anteroposterior normal que se encuentra acompañada de discrepancia dentoalveolar, malposiciones individuales y alteraciones a nivel transversal o vertical. Las alternativas de tratamiento para una Clase I con discrepancia dentoalveolar positiva son diversas; estas dependen de factores como la severidad de la maloclusión, etiología y otros, con el objetivo de obtener una estética dental y facial, buena función y estabilidad. En el caso se utilizó arcos de retracción, obteniendo resultados óptimos.

\section{Referencias}

1. Proffit W, Fields W, Sarver D. Ortodoncia contemporánea. 4ta ed. España: Editorial Mosby; 2008.

2. Villalba E, Gutiérrez DH, Díaz R, Evaluación de la relación maxilomandibular, parte clave del diagnóstico ortodóncico. Rev Latinoam Ortod y Odontoped. 2009;23(4):432-8.

3. Uribe G. Ortodoncia: Teoría y clínica, 2da ed. Colombia: Corporación para las investigaciones biológicas; 2010.

4. Proffit W. Ortodoncia. Teoría y Práctica. 2da ed. Madrid: Editorial Mosby-Doyma Libros S.A.; 1996.

5. Vellini F. Ortodoncia: Diagnóstico y planificación clínica. 2da ed. Brasil: Editorial Artes Médicas; 2004.

6. Quirós O. Bases biomecánicas y aplicaciones clínicas en Ortodoncia Interceptiva, 1ra ed. Venezuela: EditorialAmolca; 2006.

7. Angle EH. Classification of malocclusion. Dent Cosmos. 1899;41:248-64.

8. Meneses A, Mendoza F. Características cefalométricas de niños con desnutrición crónica comparados con niños en estado nutricional normal de 8 a 12 años de edad. Rev Estomatol Herediana. 2007; 17(2).

9. Ugalde F. Clasificación de la maloclusión en los planos anteroposterior, vertical y transversal. RevistaADM. 2007;64(3):97-109.

10. Alhammadi M, Halboub E, Fayed M, Labib A, El-Saaidi C. Global distribution of malocclusion traits: A systematic review. Dental Press J. Orthod. 2018 Dec; 23(6): 40.

11. López M. Determinación del índice de discrepancia dentaria en estudiantes de 4to y 5 to Año de la Carrera de Odontología de la Universidad del Valle, Gestiones 2010-2012-2013. Rev. Inv. Inf. Salud. 2015; 10(24).

12. Huancollo A. Relación entre el biotipo facial con la discrepancia alveolodentaria en pacientes de 14 a 25 años atendidos en consulta privada, Juliaca. [Tesis de grado]. Perú: Universidad Alas Peruanas; 2018.

13. Fernández CA. Estudio descriptivo de características dentomaxilares y de evaluación morfofuncional orofacial en niños entre 7 y 13 años con incompetencia labial. [Tesis de grado]. Santiago: Universidad de Chile; 2015.

14. Santoyo R, Tavira F. Tratamiento de ortodoncia acelerada en paciente con tejidos 
periodontales reducidos. Caso clínico. Rev. Mex. Ortodon. 2015; 3(2):120-127.

15. Carrera GE, Sáez EG. Tratamiento de espacios interdentales irregulares en una clase II esquelética, mediante enfoque tradicional: presentación de un caso. Rev Mex Ortodon. 2017; 5(4):260-265.

16. Lamas F. Corrección de una maloclusión Clase I mordida abierta usando dispositivos de intrusión molar. [Tesis]. Lima: Universidad Nacional Mayor de San Marcos; 2015. 